\title{
High-Sensitivity Detection of Narrowband Light in a More Intense Broadband Background Using Coherence Interferogram Phase
}

\author{
Ricardo C. Coutinho, Member, IEEE, David R. Selviah, Member, IEEE, and Hugh D. Griffiths, Fellow, IEEE
}

\begin{abstract}
This paper describes an optical interferometric detection technique, known as the interferogram phase step shift, which detects narrowband, coherent, and partially coherent light in more intense broadband incoherent background light using changes in the phase gradient with the optical path difference of the coherence interferogram to distinguish the bandwidth or coherence of the signal from that of the background. The detection sensitivity is assessed experimentally by measuring the smallest signal-to-background ratio or signal-to-clutter ratio (SCR), which causes a detectable change in the self-coherence interferogram phase. This minimum detectable SCR (MDSCR) is measured for the multimode $\mathrm{He}-\mathrm{Ne}$ laser, resonant-cavity light-emitting diode (LED), narrowband-filtered white light, and LED signal sources in a more intense tungsten-halogen-lamp white-light background. The highest MDSCRs to date, to the authors' knowledge, are

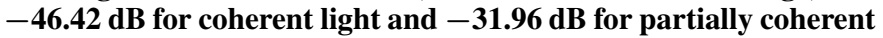
light, which exceed those of existing automatic single-domain techniques by 18.97 and $4.51 \mathrm{~dB}$ with system input dynamic ranges of 19.24 and $11.39 \mathrm{~dB}$, respectively. The sensitivity dependence on the signal-to-system bandwidth ratio and on the relative offset of their central wavelengths is also assessed, and optimum values are identified.
\end{abstract}

Index Terms-Correlators, envelope detection, gas detectors, homodyne detection, missile detection and tracking, object detection, optical signal detection, phase detection, photodetectors.

\section{INTRODUCTION}

$\mathbf{R}$ EAL-TIME detection of a weak optical signal in the presence of a more intense background signal cannot be achieved by amplifying the combined input signal, as the stronger background saturates the receiver. Special attributes of the signal that distinguish it from its background must be exploited to provide a processing gain that suppresses the background more than the signal to improve the signal-tobackground ratio or signal-to-clutter ratio (SCR). Such at-

Manuscript received September 9, 2005; revised July 3, 2006. The work of R. C. Coutinho was supported by the Brazilian Navy Weapon Systems Directorate, Rio de Janeiro, Brazil.

R. C. Coutinho is with the Brazilian Navy Weapon Systems Directorate, Rio de Janeiro 20010-000, Brazil, and also with the Department of Electronic Engineering, Federal Center of Technological Education, Rio de Janeiro 20271-110, Brazil (e-mail: riccout@yahoo.com.br; ricardo.coutinho@dsam. mar.mil.br).

D. R. Selviah is with the Department of Electronic and Electrical Engineering, University College London, WC1E 7JE London, U.K. (e-mail: d.selviah@ ee.ucl.ac.uk).

H. D. Griffiths is with the Department of Electronic and Electrical Engineering, University College London, WC1E 7JE London, U.K., and also with the Defence College of Management and Technology, Cranfield University, Shrivenham, SN6 8LA, U.K. (e-mail: h.griffiths@ee.ucl.ac.uk).

Digital Object Identifier 10.1109/JLT.2006.881851 tributes of the signal include the wavelength, intensity, angular divergence, spatial power density or divergence, temporal modulation, polarization, and phase or shot noise. Intensity alone is insufficient, as the signal is hidden beneath the stronger background. In this paper, we make use of the bandwidth or coherence of the signal as compared to that of its background. The bandwidth of a signal is inversely proportional to its coherence length and is independent of its center wavelength and phase. The highest contrast is achieved when the signal has the narrowest bandwidth or linewidth and the longest coherence length as in a laser and when the background has the widest bandwidth and shortest coherence length as in white light. The bandwidth of a modulated narrow-linewidth laser is determined by the modulation frequency directly or via laser chirp.

Applications of such a system include use in the detection of laser guide beams or sights aimed from the direction of the sun, a modulated free-space optical communication beam in the presence of a more powerful broadband jamming signal, hot glowing gasses and cold gas absorption lines [1] in sunlight, a reflecting object in a broadband illuminated scene, a deliberately buried quantum cryptographic signal beneath an ambient noise floor, a one-wavelength channel among multiple adjacent wavelength-division multiplexing (WDM) wavelength transmission channels, and a fluorescing cell in a strongly scattering background. However, it cannot be used for spreadspectrum communications since each signal is spread over the same bandwidth.

An interferometer [2] that is placed before a detector finds the bandwidth or coherence length of the combined signal by interfering the signal with a delayed version of itself. The broadband background has a short coherence length, and when the narrowband signal is also present, the combined signal has increased coherence length. However, the coherence length is difficult to establish consistently, as the background signal degrades the fringe visibility in the interferogram for large delays. To improve the minimum detectable SCR (MDSCR), the interferometer is preceded by a bandpass filter, which suppresses most of the broadband background. The filter must have sufficient bandwidth to receive the signal whose wavelength may not be precisely known. In addition, the filter plays an important role in the signal processing, "using the background signal itself" to substantially improve the sensitivity when a rectangular bandpass filter [3] is used.

The earliest experimental measurements using this method with a laser source gave the best detection sensitivities, but the experimental conditions were unclear [4], and the system was 
(a)

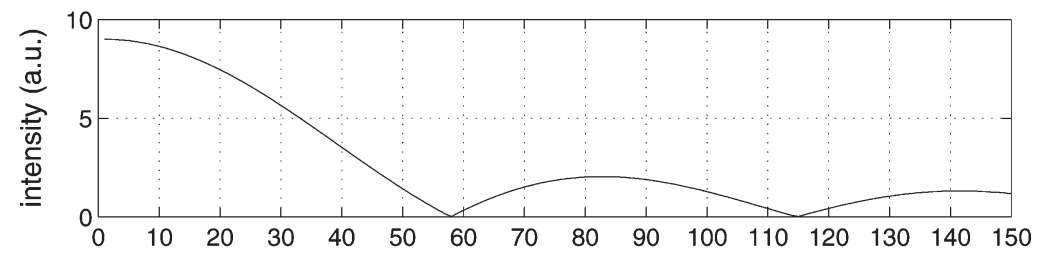

(b)

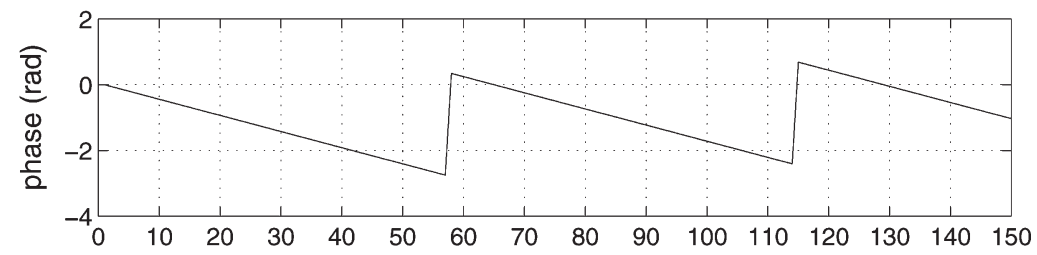

(c)

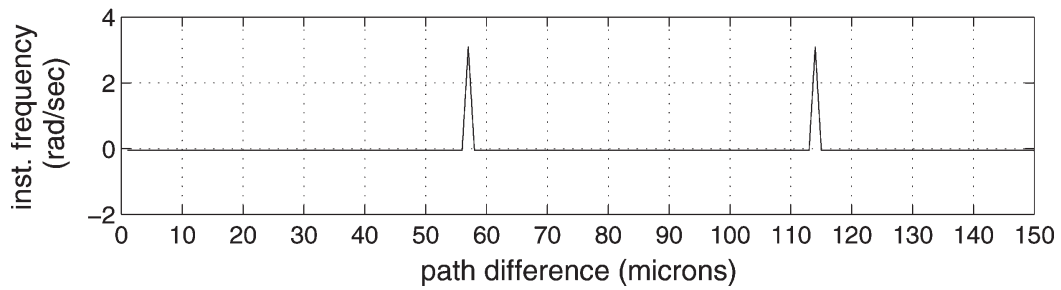

Fig. 1. Theoretical ideal interferogram due to an input rectangular filter (a) absolute amplitude, (b) phase, and (c) instantaneous frequency.

not automatic, as someone was required to view an oscilloscope trace and to recognize when a feature was displaced [5]. Experimental demonstrations using partially coherent sources gave poorer detection sensitivities [5]-[7].

In Section II, we describe a postdetector signal processing algorithm, known as interferogram phase step shift (IPSS), to optimize the receiver sensitivity and discuss its relationship to a similar technique, known as Fourier transform spectroscopy (FTS) [8], and to earlier similar works. The receiver's sensitivity is characterized experimentally with the system described in Section III, using the laser, resonant-cavity light-emitting diode (RCLED), narrowband-filtered white light, and LED sources in Section IV and the monochromator sources in a strong tungsten-halogen-bulb white-light background in Section V. The optimum filter bandwidth and wavelength relative to the source wavelength are established in Sections V and VI.

\section{IPSS DETECTION PRINCIPLES}

In the IPSS method, a rectangular passband input filter creates a "localized feature" in the "gradient of the phase" of the coherence interferogram. We then scan this localized feature to note changes in its position in the optical path difference, which depends on the net degree of coherence of the combined input signal. The update rate is therefore fast suitable for rapidly changing applications.

In FTS, the self-coherence interferogram [2] of the light that enters the predetector interferometer is found by scanning the optical path difference between the signal and a delayed version of itself through a large range that is comparable to the coherence length of the signal, which may be centimeters for lasers, as the spectral resolution achieved from the Fourier transform is the reciprocal of the length of the scan [8]. The time required for the long FTS scan followed by a Fourier transform poses a limitation in real-time applications that require high update rate. In the IPSS detection method, only a small range of optical path differences is repeatedly scanned without sacrificing spectral resolution and with high update rate. In FTS, a reference laser is required for the path difference calibration of the long scan, but in IPSS, it is not required because the absolute path difference value is not needed. Nonlinear scaling of the path difference due to nonlinear translation stages is also less important in IPSS than in FTS, where it distorts the phase before Fourier transformation. Insufficient collimation of the input light causes an extra path difference proportional to the scan length [8], which is more serious in FTS than in IPSS due to its much longer scan length.

The IPSS method uses an almost rectangular bandpass input optical filter with a steep rolloff. We assume that the background spectrum is substantially flat within the filter's bandwidth, which is often the case for broadband sources. Then, since the self-coherence function is the Fourier transform of the power spectrum [2], it has a $\operatorname{sinc}(\sin (x) / x)$ function shape. Fig. 1(a) shows the theoretical envelope of the absolute value of the inverse Fourier transform of a rectangular filter response. Fig. 1(b) shows the calculated phase of the interferogram fringes within the envelope, and Fig. 1(c) shows the instantaneous frequency of the fringes, which is obtained by differentiating the phase. The phase has $-180^{\circ}$ steps at the positions of the nulls. We select the first null of the sinc function, as its position is very sensitive to the input coherence and it has a higher signal-to-noise ratio (SNR) than the other nulls. In addition, following [3], we choose the phase step of this null rather than its amplitude minimum since its position 


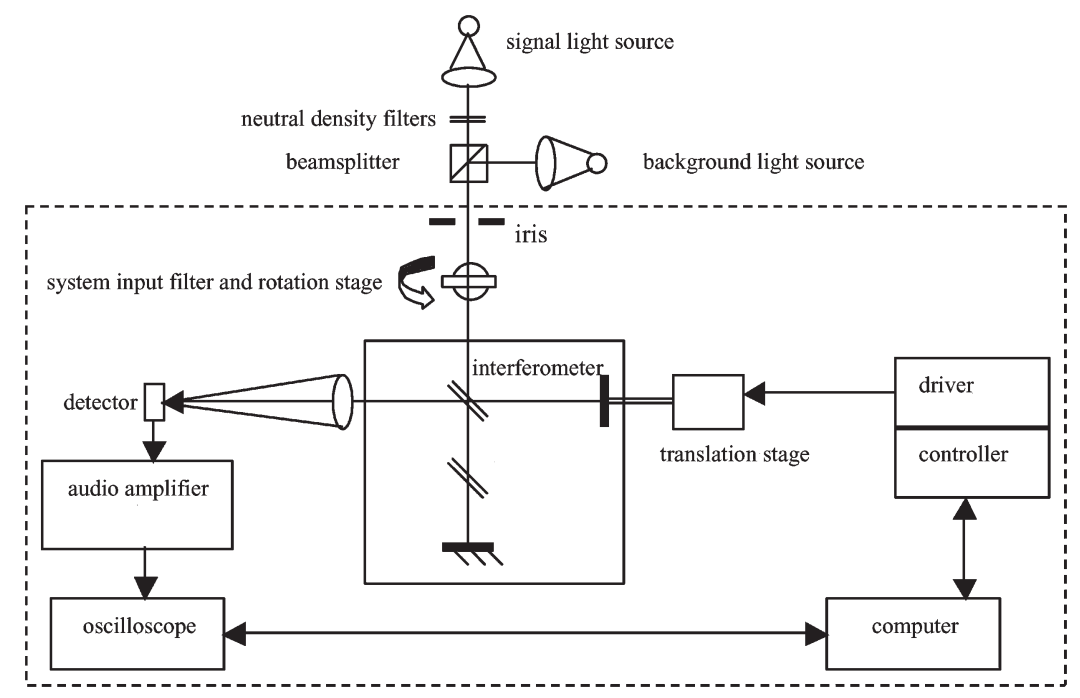

Fig. 2. Experimental arrangement.

is easier to define accurately; as in this region, fringe visibility is very low and is degraded by additive noise due to, e.g., the receiver photodetector.

In this paper, we go beyond the earlier work [3] and improve the sensitivity by differentiating the phase and using the instantaneous frequency instead of the phase, as this removes the slope of the phase function so that we can use a simple amplitude threshold [Fig. 1(b) and (c)]. Furthermore, as we are interested in sudden phase changes, the differentiation acts as a high-pass filter, increasing the relative size of these changes and discarding low-frequency variations.

The position of the frequency spike due to the first minimum of the interferogram envelope is recorded when only the background signal is present to give a reference value, with which subsequent scans can be compared. When a coherent source moves into the field of view, its narrow spectral feature increases the net degree of coherence of the scene, moving the interferogram first minimum's frequency spike to higher path differences. If the narrow spectral feature is a gas absorption line, the displacement of this frequency spike will be toward lower path differences. By tracking the shift of the first null's frequency spike, detection can be declared whenever this shift is larger than a specified threshold. Unusually, the detection signal is the "displacement" of the frequency spike due to the presence of the coherent light at the input, and the noise is all the other displacements, such as those due to changes in the background spectrum.

\section{IPSS EXPERIMENTAL METHOD}

The visible band was selected for the experiment (Fig. 2) to aid optical component alignment. An optical table floated with compressed gas isolated the system from vibrations. The detection system is shown in the dotted rectangle in Fig. 2, and the elements outside simulate the combined background and signal sources. A $20-\mathrm{W}$ tungsten halogen lamp with a blackbody temperature of $3220 \mathrm{~K}$ was used as the broadband background light. The light bulb was placed in an enclosure with a concave rear mirror, a front aperture stop, and a 30-mm $\mathrm{f} / 2$ plano-convex lens to collimate its beam. Due to the size of the bulb's filament, the degree of collimation of the signal and the background differed, but this had little impact on the experimental results due to the small scan length [8]. Neutral density filters were placed in the collimated signal path to give different signal-to-background ratios or SCRs. The light from the two sources was combined in a nonpolarizing cube beam splitter before entering the detection system.

The light exiting the beam splitter was spatially limited by an iris (Fig. 2) to allow better control of the SCR. The iris diameter limited the beam diameter to that of the components and apertures of the system so that the light measured at the system's entrance reached the detector. The 28-mm-diameter interferometer mirrors had the smallest width of the optical components along the beam path. A reference plane was defined after the iris and the interference filter, where power measurements were taken using the system photodetector to establish the filtered SCR. The same plane was used when the interference filter was removed to establish the unfiltered SCR values. The unfiltered background power was in the range of $32.5-145.5 \mu \mathrm{W}$. The filter reduced the background signal by $18.68 \mathrm{~dB}$. The interference filter was selected depending on the wavelength of the signal to be detected. The multilayer filters [2] had fast rolloff, as required by the IPSS method and, because of their directional characteristics, were mounted on a rotation stage so that maximum transmission could be achieved. The filtered beam entered a Michelson interferometer, which gave the coherence interferogram as a function of the path difference. The input beam was split into two and traveled different length paths. The path difference was determined by a mirror mounted on a dc-motorized translation stage with a resolution of $0.1 \mu \mathrm{m}$, which allowed it to be scanned longitudinally normal to its surface. This stage was controlled from a LabVIEW program running on a computer via a Klinger MC4 controller. The speed of the stage, the length of the scan, and the number of scans to be averaged were programmed. A $10-\mu \mathrm{m}$ scan length, corresponding to a $20-\mu \mathrm{m}$ path difference range, was chosen, and this was scanned at a speed of $150 \mu \mathrm{m} / \mathrm{s}$ in steps of $0.1 \mu \mathrm{m}$. The speed of the scan gave a mean fringe frequency in the range of 


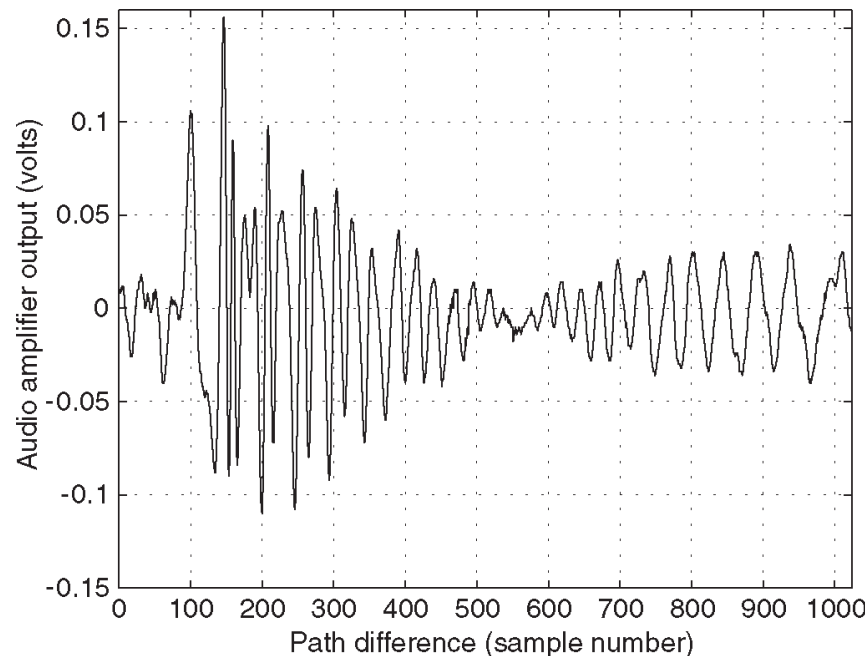

Fig. 3. Part of an interferogram from a tungsten-halogen-bulb light source filtered by a narrowband filter that was acquired using the translation stage.

$150-160 \mathrm{~Hz}$ for the wavelengths used $(630-680 \mathrm{~nm})$. The mirror scanned at around $2 \mathrm{~Hz}$. The translation stage suffered from hysteresis, so only one of the directions of the scan was used.

A plano-convex lens collected and focused the light from the interferometer's exit aperture onto a $1-\mathrm{cm}^{2}$ detector. In Fig. 3, the first null is clearly visible where the signal amplitude is minimum and has the lowest SNR and fringe visibility. To obtain sufficient SNR, an EG\&G Brookdeal 9452 audio amplifier amplified the fringe signals from the detector by $30 \mathrm{~dB}$. The amplifier also had a built-in analog bandpass filter, whose 3 -dB cutoff frequencies were set to $100 \mathrm{~Hz}$ and $10 \mathrm{kHz}$ to limit the noise bandwidth. The amplifier's output was acquired by a Tektronix 2430A digital oscilloscope, which removed the dc level by ac coupling at a sampling frequency of $5.12 \mathrm{kHz}$ and stored interferogram scans (Fig. 3) as ASCII 1024 sample data files. The noisy region at the left of Fig. 3 was caused by the random oscillations of the mirror when the stage reversed. This artifact was removed by cropping all of the interferograms at the same path difference. In a practical real-time system, this could be performed by gating the input.

Several interferograms acquired in successive scans were averaged together to further suppress noise. In order to align the interferograms for correct averaging and for comparison with the reference interferogram, the mirror scan was synchronized to the oscilloscope scan by triggering the oscilloscope with a signal generated by the motor controller at the start of each scan. The 35-ms delay before the translation stages moved had no effect on the results as the full interferogram was acquired. The clarity of the instantaneous frequency spike depended on the number of scans averaged and on the initial SNR. If insufficient interferograms were averaged, a reduced peak was obtained, and if too many were averaged, the peak was removed due to the low-pass filtering effect of the averaging process.

Fig. 4 shows a typical experimental waveform at each stage of processing by the IPSS algorithm. It is in good agreement with the theoretical ideal of Fig. 1. Two waveforms are seen in each plot: The upper waveform has the signal present, while the lower reference has no signal present. Fig. 4(a) shows the raw signals recorded, whereas Fig. 4(b) shows those after they were filtered using a software filter and averaged. In both cases, the shift of the first-null feature, as the net coherence increases when the signal is present, is clear. The reference trace was taken immediately before the signal trace, and the two traces were compared to detect the presence of the signal source. This removed effects such as temperature and temporal drift of the components. Fig. 4(c) shows the unwrapped phase with $180^{\circ}$ phase steps, and Fig. 4(d) shows the instantaneous frequency with very clear noiseless narrow spikes marking the position of the first null of the interferogram. The clarity of the instantaneous frequency spikes produced by the IPSS signal processing is the key to obtaining high MDSCR.

The MDSCR was measured for a coherent helium-neon (He-Ne) gas laser optical source and partially coherent RCLED, filtered white light, and LED sources. Due to the divergence of the partially coherent sources, they were collimated by a $30-\mathrm{mm} \mathrm{f} / 2$ plano-convex lens with an iris aperture stop. For the partially coherent sources, an input filter having a similar central wavelength was selected, and its central wavelength was tuned by rotating the filter to maximize the transmitted power. An input iris and an output focusing lens were found to improve the SNR, so they were used for all sources, except the filtered white light and the LED sources. The experimental parameter values used in these experiments are summarized in Table I.

\section{IPSS RESULTS}

\section{A. He-Ne Gas Laser Source}

A multimode 5-mW unpolarized Melles Griot 05 LLR $851 \mathrm{He}-\mathrm{Ne}$ red laser was switched on and left to operate for $1 \mathrm{~h}$ before any measurements were taken to allow its modes to stabilize. Its beam was expanded by a $\times 10$ microscope objective, which is slightly tilted relative to the laser exit aperture to avoid reflections back into the laser, which would cause intensity fluctuations. Spatial filters were not required to select a single spatial mode, as we found that the reduction in coherence length of the laser, due to its multiple transverse modes, did not prevent detection, which is a benefit of the IPSS technique. The laser's spectral line had four or five Lorentzian longitudinal modes [9] under a Gaussian Doppler-spreading envelope. The expanded laser beam was collimated by a double achromatic lens. The unfiltered laser power was in the range of $0.935 \mathrm{nW}-2.77 \mu \mathrm{W}$.

Fig. 5 shows the displacement of the first-null phase step as a function of input filtered SCR. Little or no displacement in the phase step occurred until the filtered SCR reached $-30 \mathrm{~dB}$, where the curve clearly departed from zero and the presence of the laser is detected. We defined the system's MDSCR to be the smallest SCR at which the error bars no longer touch zero [10], [11]. The detection dynamic range (Table II) was limited at the other extreme at an SCR of $-8.5 \mathrm{~dB}$, where the signal amplitude was so strong that the phase step no longer occurred.

\section{B. RCLED Source}

An RCLED with coherence lengths [12] in the range of 22-32 $\mu \mathrm{m}$ has a coherence length that depends on numerical aperture (NA), so it was mounted on an optical rail, as in [12], using a microscope objective having a nominal NA set 

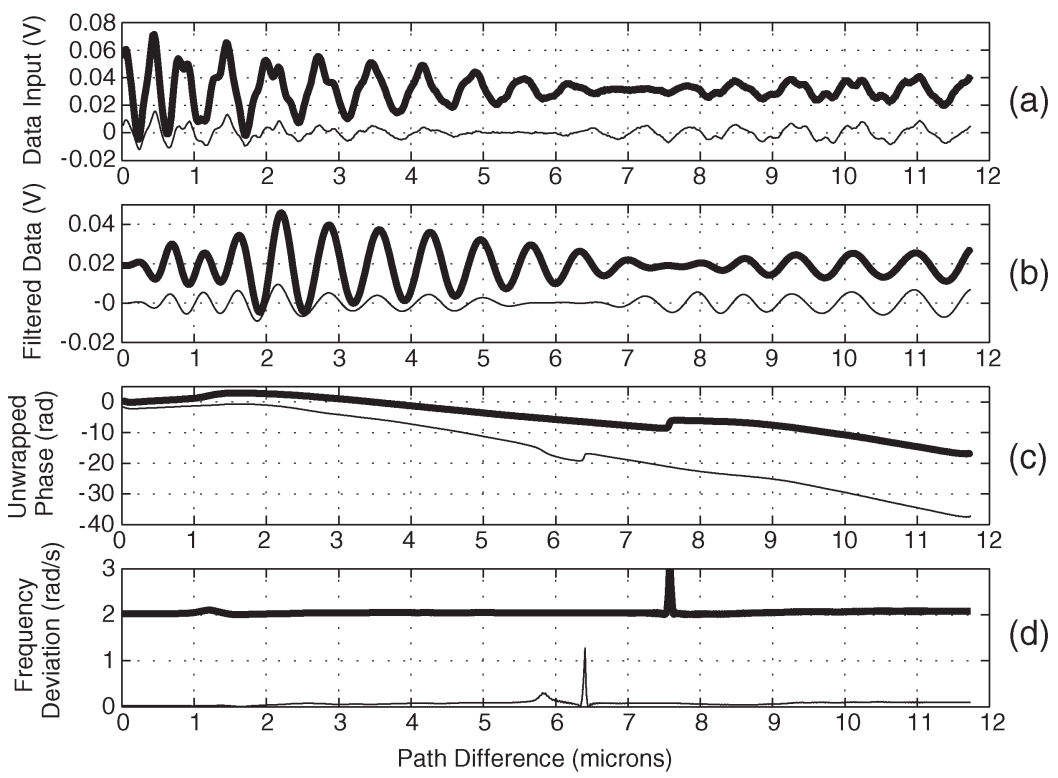

Fig. 4. Typical experimental waveforms obtained during the IPSS signal processing. (a) Raw interferogram signal. (b) Software-filtered and averaged interferogram signal. (c) Phase of carrier. (d) Instantaneous frequency of carrier. The bold trace has a signal, whereas the normal reference has no signal present.

TABLE I

EXPERIMENTAL PARAMETER VALUES FOR MDSCR MEASUREMENTS

\begin{tabular}{ccccccccc}
\hline \hline Signal & $\begin{array}{c}\text { Signal } \\
\text { central } \\
\text { source }\end{array}$ & $\begin{array}{c}\text { Signal } \\
\text { BW }\end{array}$ & $\begin{array}{c}\text { Signal } \\
\text { coh. }\end{array}$ & $\begin{array}{c}\text { Filter } \\
\text { central } \\
\text { FWH }\end{array}$ & $\begin{array}{c}\text { Filter } \\
\text { length }\end{array}$ & $\begin{array}{c}\text { BW } \\
\text { FWHM }\end{array}$ & $\begin{array}{c}\text { Signal } \\
\text { /filter }\end{array}$ & \multicolumn{2}{c}{$\begin{array}{c}\text { No. of } \\
\text { BW } \\
\text { ratio }\end{array}$} & $\begin{array}{c}\text { I/P iris } \\
\text { ave. }\end{array}$ & lens \\
& $\mathrm{nm}$ & $\mathrm{nm}$ & $\mu \mathrm{m}$ & $\mathrm{nm}$ & $\mathrm{nm}$ & & & \\
\hline Laser & 632.8 & 0.002 & 200000 & 632.8 & 11 & 0.0002 & 4 & $\sqrt{ }$ \\
RCLED & 650 & 10 & 22.77 & 651.9 & 36.2 & 0.2762 & 1 & $\sqrt{ }$ \\
Filtered & 648.7 & 12.2 & 11.48 & 651.9 & 36.2 & 0.3370 & 20 & $\times$ \\
LED & 644 & 18 & 5.44 & 651.9 & 36.2 & 0.4972 & 5 & $\times$ \\
\hline \hline
\end{tabular}

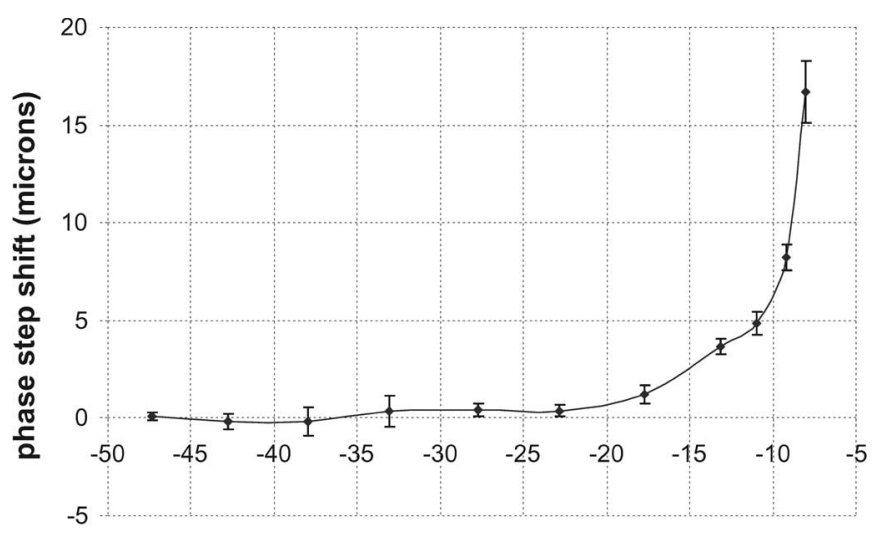

filtered signal-to-clutter ratio (dB)

Fig. 5. Displacement of the phase step at the first null of the interferogram for a He-Ne laser source as a function of the SCR after the input filter.

at 0.65 , which is its maximum. Different SCRs were obtained by varying the device's supply current from 0 to $40 \mathrm{~mA}$. The bandwidth of the RCLED varied by only $3 \%$ over this range of driving currents, so this variation was neglected. The unfiltered input RCLED power was in the range of $20-67 \mu \mathrm{W}$. The microscope objective was adjusted to give an image of the RCLED on the detector.
TABLE II

EXPERIMENTAL RESULTS SUMMARY

\begin{tabular}{cccccc}
\hline \hline Signal & $\begin{array}{c}\text { Standard } \\
\text { deviation } \\
\text { /average }\end{array}$ & $\begin{array}{c}\text { Filtered } \\
\text { MDSCR } \\
\mathrm{dB}\end{array}$ & $\begin{array}{c}\text { Unfiltered } \\
\text { MDSCR } \\
\mathrm{dB}\end{array}$ & $\begin{array}{c}\text { Max. } \\
\text { phase } \\
\text { step shift } \\
\mu \mathrm{m}\end{array}$ & $\begin{array}{c}\text { Dynamic } \\
\text { Range } \\
\mathrm{dB}\end{array}$ \\
\hline Laser & 1.05 & -27.74 & -46.42 & $16.7 \pm 1.6$ & 19.24 \\
RCLED & 0.21 & $<-11$ & $<-30$ & $6.8 \pm 1.1$ & $>4.7$ \\
Filtered & 1.7 & -20.39 & -31.96 & $2.9 \pm 2.0$ & 11.39 \\
LED & 2.5 & -5.85 & -13.07 & $1.06 \pm 0.4$ & 16.1 \\
\hline \hline
\end{tabular}

The SCR was measured at the detector plane due to lack of space to position the detector at the entrance of the interferometer after the interference filter. The SCR at the detector plane is the same as that at the interferometer entrance if the optical transmission through the interferometer is the same for the filtered signal and the background light. This was confirmed by measuring the SCRs at the input and output after filtering by the interference filter, due to the relatively narrow optical bandwidth of both light sources and due to similar degrees of collimation. Fig. 6 shows the MDSCR.

\section{Narrowband-Filtered Tungsten-Halogen-Bulb White-Light Source}

A tungsten-halogen-bulb white-light source filtered by an interference filter gave signal powers in the range of $39.9 \mathrm{nW}-10.5 \mu \mathrm{W}$ before the system filter. Fig. 7 shows the noise due to intensity fluctuations of both the background and the source tungsten halogen bulb, which are caused by the $50-\mathrm{Hz}$ supply.

\section{LED Source}

An ultrabright InGaAlP 15000 mcd LED signal source power was controlled by changing its supply current from 0 to $40 \mathrm{~mA}$ (Fig. 8). 


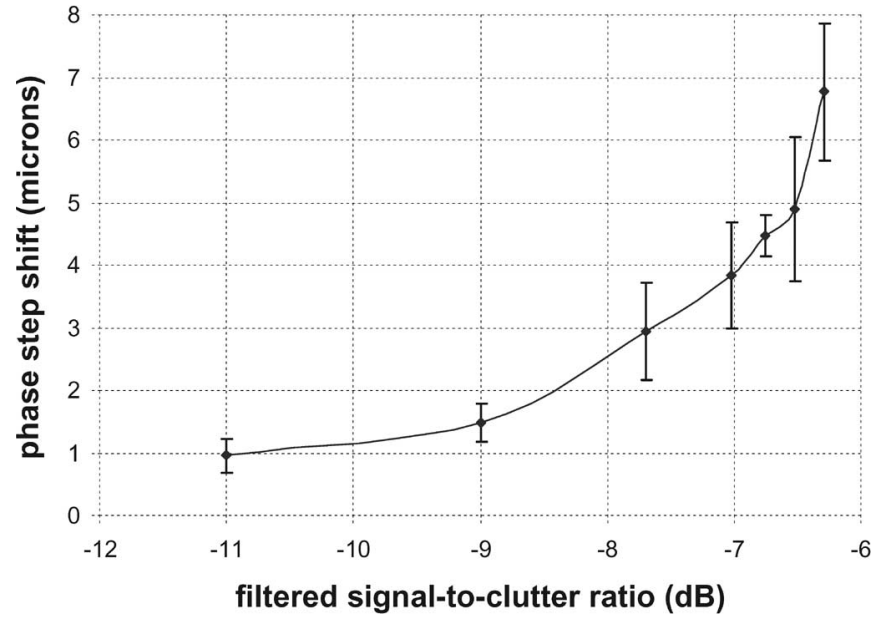

Fig. 6. Displacement of the phase step at the first null of the interferogram for an RCLED as a function of the SCR after the input filter.

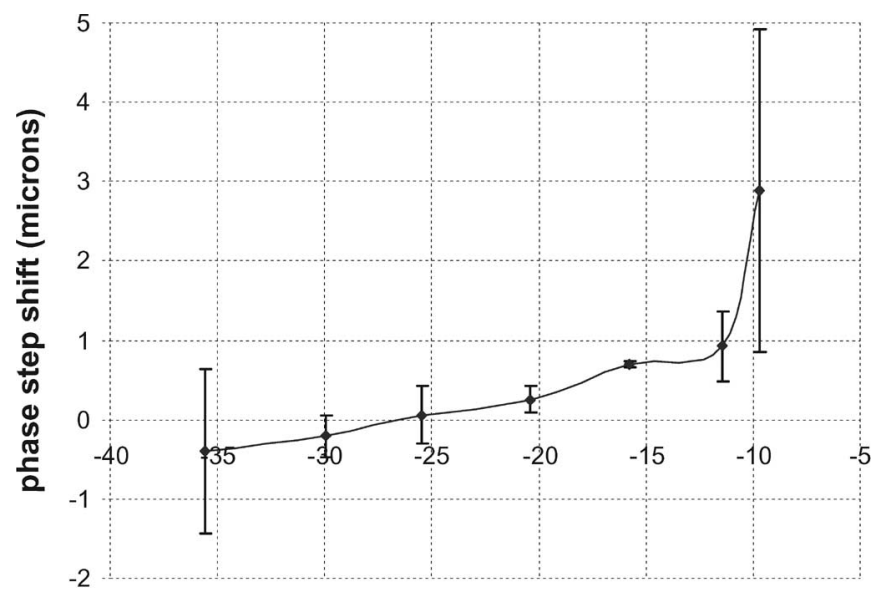

filtered signal-to-clutter ratio (dB)

Fig. 7. Displacement of the phase step at the first null of the interferogram for a 12.2-nm-bandwidth filtered light source as a function of the SCR after the input filter.

\section{E. Discussion}

Table II summarizes the results of Figs. 5-8. The MDSCR for the laser shows that the detection method is remarkably sensitive to coherent sources. The laser's phase noise was converted to relative intensity noise by the interferometer and was included in the measurements. The noise given by the standard deviation divided by the average value for the displacements was the lowest for the RCLED, showing that it had the most stable output. The zero-displacement crossing was not obtained in Fig. 6, so only an upper bound on MDSCR and lower bound on dynamic range could be given. The noise for the filtered white-light source was larger than that for the laser due to its ac supply; nevertheless, a very sensitive MDSCR was obtained, although it was not as good as that for the laser, which had the narrowest spectral linewidth. The LED had the largest noise and largest bandwidth and gave the poorest sensitivity. The system's input dynamic range was fairly consistent, being slightly higher for the coherent laser source.

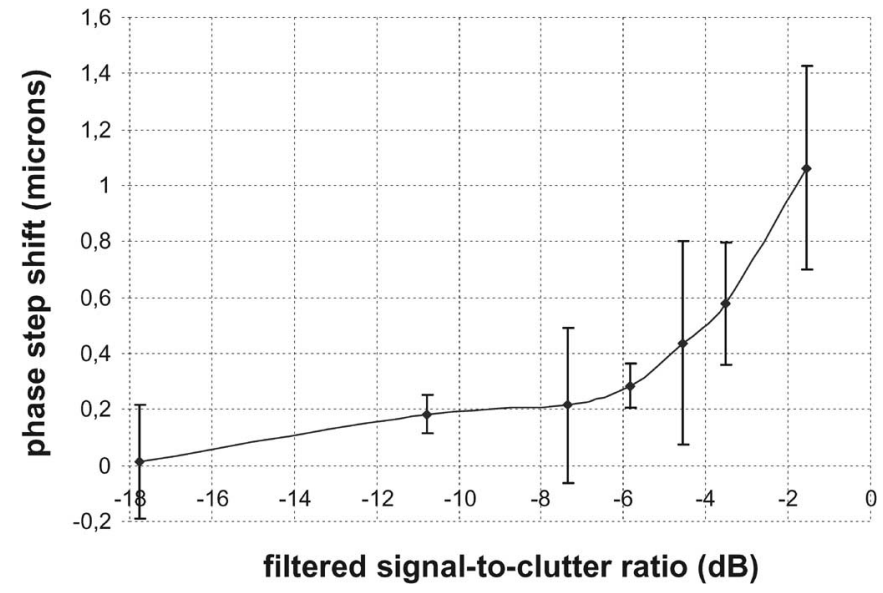

Fig. 8. Displacement of the phase step at the first null of the interferogram for a LED as a function of the SCR after the input filter.

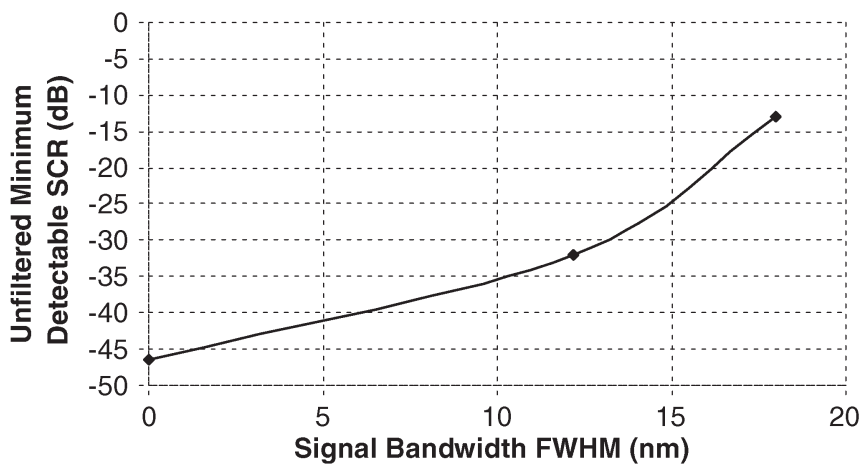

Fig. 9. MDSCR as a function of the source bandwidth for a He-Ne laser, filtered tungsten-halogen-bulb light, and LED sources.

Fig. 9 shows the dependence of the MDSCR on source bandwidth. The RCLED results were omitted, as only an upper bound was found. The MDSCR sensitivity is clearly directly dependent on source bandwidth as expected since this is the criterion used to distinguish the source from the background.

\section{Signal-To-Filter Bandwidth Ratio (BWR)}

Experiments were performed to establish the optimum input filter's bandwidth for the best MDSCR. An optical signal source whose central wavelength and bandwidth could be easily tuned was constructed by passing light from a powerful $250-\mathrm{W}$ tungsten-halogen-bulb white light with a blackbody temperature of $3200 \mathrm{~K}$ through a Bentham M300 grating monochromator filter with a dispersion of $1.8 \mathrm{~nm} / \mathrm{mm}$. Our approach is similar to that in [13]; however, we set both the input and output monochromator slits to a similar width of $3 \mathrm{~mm}$ and fully illuminate the input slit, so the bandwidth was given by the product of the slit width and the grating dispersion, resulting in a bandwidth of $5.4 \mathrm{~nm}$. A periscope matched the beam heights of the monochromator and the interferometer, and a plane-convex lens collimated the beam. The beam diameter was limited to $10 \mathrm{~mm}$ by the input iris. 
TABLE III

SigNAL/FILTER EXPERIMENTAL COMBINATIONS

\begin{tabular}{ccccc}
\hline \hline Set & $\begin{array}{c}\text { Central } \\
\text { wavelength } \\
\mathrm{nm}\end{array}$ & $\begin{array}{c}\text { Signal } \\
\text { bandwidth } \\
\mathrm{nm}\end{array}$ & Filter bandwidth & $\begin{array}{c}\text { Signal to filter } \\
\text { bandwidth ratio }\end{array}$ \\
\hline \hline 1 & 651.9 & 5.4 & 36.2 & 0.1492 \\
2 & 674.8 & 5.4 & 17.8 & 0.3034 \\
3 & 632.6 & 5.4 & 11 & 0.4909 \\
\hline \hline
\end{tabular}

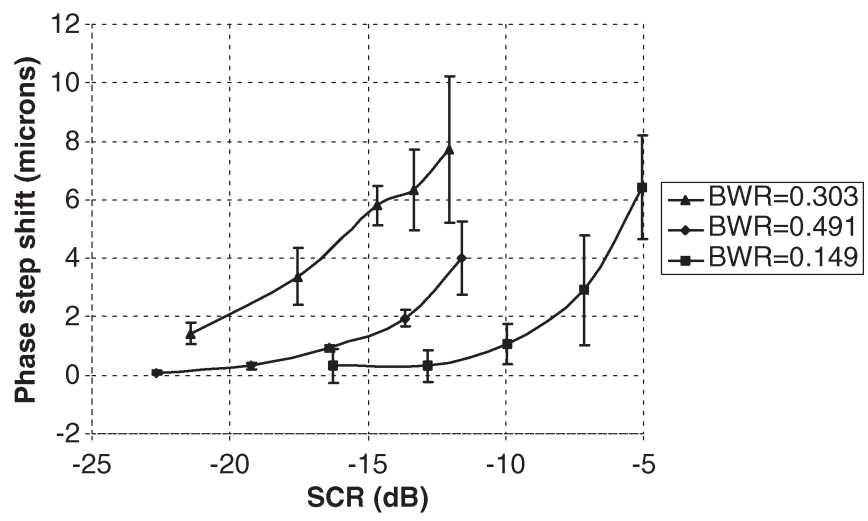

Fig. 10. Displacement of the phase step at the first null of the interferogram for several source-to-input-filter BWRs listed in Table III as a function of the filtered SCR.

The detection system was the same as that in Section IV but with bandpass filtering in the audio amplifier between $100 \mathrm{~Hz}$ and $1 \mathrm{kHz}$ and an oscilloscope sampling frequency of $10.24 \mathrm{kHz}$. Different SCRs were obtained by attenuating the radiation from the signal using different power settings for the $250-\mathrm{W}$ signal light source and attenuating the background using neutral density filters. This slightly different arrangement was due to the low monochromator throughput giving very low signal power at the interferometer entrance. Hence, instead of placing neutral density filters in the signal path, these were placed in the background path.

Three signal-bandwidth-to-input-filter-BWR combinations were used (Table III). The monochromator wavelength was chosen to give signals with central wavelengths coincident with those of the available interference filters. As the coherence length is proportional to the wavelength squared [2], this procedure slightly varied the coherence length of the signal, but as the central wavelength variation between the three filters is small $( \pm 3 \%)$, this effect was considered to be negligible. For each signal/filter combination, five different power ratios were tested, and five to eight null feature displacements were combined to give an average and a standard deviation per point.

Fig. 9 shows the phase step displacement as a function of the filtered SCR for each of the signal/filter combinations. The optimum MDSCR of $<21.5 \mathrm{~dB}$ would occur for a signalto-filter BWR of 0.303 , similar to that of the filtered light source used in Section IV if the curve were extended to the zero axis in Fig. 10.

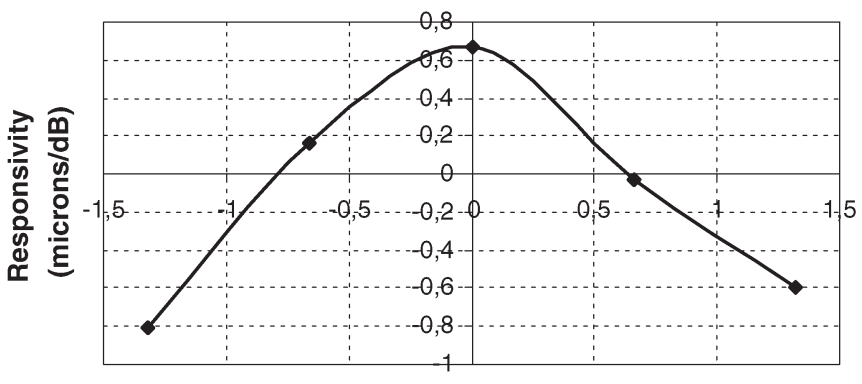

Central wavelength offset ( $\%$ of filter bandwidth)

Fig. 11. Responsivity as a function of the central signal and filter wavelength offset.

\section{Signal-to-Filter Central Wavelength Offset}

In the earlier experiments, we aligned the input filter's central wavelength with the signal's central wavelength after selecting the most similar filter by rotating them to tune the filter's wavelength to maximize the power transmitted; however, this does not ensure that the central wavelengths are coincident, so we investigated the effect of relative wavelength offsets on the detection sensitivity. As the MDSCR did not vary significantly over the range of signal-to-filter wavelength offsets studied, we instead observed the gradient of the phase step displacement versus SCR curve near the MDSCR, which we call the responsivity (in micro meters per decibel), in order to distinguish them.

The filter with a BWR of 0.3 in set 2 in Table III was used, as it gave the best MDSCR in the earlier aligned wavelength experiment. Different signal central wavelengths (665.9, $670.35,674.8,679.25$, and $683.7 \mathrm{~nm}$ ) were achieved by turning the monochromator grating. Fig. 11 plots the responsivity as a function of the relative central wavelength offset between the signal and the input filter. The responsivity is most positive when the wavelengths are the same. The responsivity reduces to zero at central wavelength offsets of $\sim 0.7 \%$ of the filter bandwidth. Further offsets cause the responsivity to become negative and of almost the same magnitude as that when the wavelengths are aligned. In this region, the signal adds to the rolloff of the filter, making the overall bandwidth larger, so the net coherence of both reduces. The positive responsivity is reduced if the signal central wavelength is not accurately centered in the optical filter spectral response, so previous knowledge of the signal spectral characteristics will allow the filter to be configured for the greatest responsivity. It remains to be established whether the optimum signal-to-background BWR remains optimum as the offset increases.

In order to make sure that the loss of responsivity is due to the offset and not the reduction of signal power coupled through the optical filter, the signal power was increased when the offset was maximized to maintain the transmitted signal power through the filter. The negative displacement of the phase step increased until the observability cutoff was reached, confirming that the negative responsivity was not due to a reduction in the filtered signal power.

Fig. 12 shows an interferogram recorded with a signal of a central wavelength of $683.7 \mathrm{~nm}$ and a filter centered at $674.8 \mathrm{~nm}$. The plot shows a beating effect that arises from the 


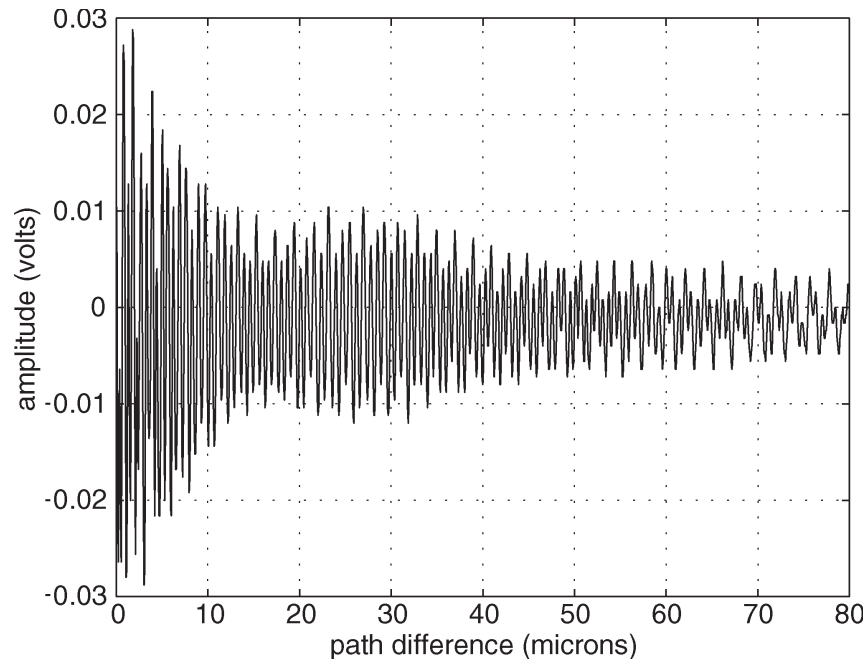

Fig. 12. Coherence interferogram showing the beating effect arising from an offset between the central wavelengths of the signal and the input filter.

wavelength offset between the signal and the filter, which is visible as an additional modulation on the fringe carrier.

\section{CONCLUSION}

This paper describes and assesses the minimum detectable signal-to-background ratio or the MDSCR of a signal processing technique, known as IPSS, for a range of coherence-length sources, including laser, RCLED, filtered tungsten-halogenbulb white light, LED, and monochromator-filtered tungstenhalogen-bulb white light. The phase step or instantaneous frequency spike displacement versus SCR after input filtering curves were recorded, showing that the MDSCR improves for narrower signal bandwidths, as expected. We converted the results of earlier works to an equivalent MDSCR in order to compare with our results, making reasonable assumptions [14]. In the earliest experiments, the use of an input filter and interferometer to detect the presence of a laser [4], [5] obtained an MDSCR of $-51.8 \mathrm{~dB}$, but these were not automatic systems. The best result of earlier experiments using an input filter and interferometer to detect partially coherent light gave an MDSCR of $-27.45 \mathrm{~dB}$ [7]. Montgomery et al. [15] gave an excellent MDSCR of $-43.8 \mathrm{~dB}$ by combining two-band spectral FTS with spatial and temporal filtering. However, temporal FTS alone has achieved the best MDSCR of $-21.8 \mathrm{~dB}$ [16]. Since our IPSS technique can also be combined with spatial and temporal filtering in the future to further improve the sensitivity, it should be compared to the single-domain approaches. Therefore, IPSS has an MDSCR of $-46.42 \mathrm{~dB}$ for the detection of coherent light and an MDSCR of $-31.96 \mathrm{~dB}$ for the detection of partially coherent light, which are better by 18.97 and $4.51 \mathrm{~dB}$, respectively, than other automatic singledomain techniques, to date, to our knowledge [7].

The experimental limitations included the degree of light collimation, the accuracy of measurement of the phase step displacement, which is caused by a limited repeatability of the translation stage of $0.1-0.6 \mu \mathrm{m}$, and $100-\mathrm{Hz}$ intensity fluctuations, which are caused by the $50-\mathrm{Hz}$ ac-driven tungsten halogen lamps used for the background and filtered signal light.
The filter reduced the signal, in addition to the background in the case of wider bandwidth signals, the filtered tungsten halogen light, and the LED, as shown by comparing the filtered and unfiltered MDSCRs, although the filter bandwidth was chosen to be larger than that of the signals. The bandwidth of the RCLED varied by $3 \%$ across the range of driving currents, which was neglected, and the LED was assumed to have a constant bandwidth that is independent of the driving current.

The dynamic range was found to be $19.24 \mathrm{~dB}$ for lasers and $11.39 \mathrm{~dB}$ for partially coherent filtered light. Input adaptive gain control could adjust the signal to lie within the dynamic range.

The signal-to-filter BWR was found to have an optimum value of $\sim 0.3$. There was a reduction in responsivity and even a reverse in the displacement direction if the filter was not aligned to the wavelength of the signal source.

These measurements were performed in the laboratory and did not include the effects due to the propagation of the optical beams in the atmosphere or vibration of the system. The susceptibility of the IPSS approach to these effects should be established by field experiments.

\section{ACKNOWLEDGMENT}

The authors would like to thank D. Wickramasinghe, Dstl Portsdown West, for providing some of the experimental apparatus, Prof. H. French for invaluable discussions, and the Brazilian Navy for granting R. C. Coutinho a sabbatical leave to take part in this paper.

\section{REFERENCES}

[1] R. D. Hudson, Infrared System Engineering. New York: WileyInterscience, 1969.

[2] E. Hecht, Optics, 3rd ed. New York: Addison-Wesley, 1998.

[3] H. A. French and P. Sutton, "Electrical signal event marker circuit," U.K. Patent 2261727A, 1993.

[4] H. A. French, "Introduction to optical transform image modulation techniques and their application to ESM data processing," in Proc. Inst. Elect. Eng. Colloquium Signal Process. ESM Syst., 1988, pp. 9/1-9/2.

[5] C. J. Duffy and D. Hickman, "A temporal coherence-based optical sensor," Sens. Actuators, vol. 18, no. 1, pp. 17-31, 1989.

[6] D. Hickman and C. J. Duffy, "Incorporation of imaging into a temporal coherence sensor," Opt. Lett., vol. 13, no. 10, pp. 898-900, Oct. 1988.

[7] S. M. Drum, "The remote detection of gases using coherence measurement," Ph.D. dissertation, Univ. Southampton, Southampton, U.K., 1990.

[8] J. E. Chamberlain, The Principles of Interferometric Spectroscopy. New York: Wiley-Interscience, 1979.

[9] "Melles Griot product catalogue," Helium Neon Lasers Data Sheet, 1999. pp. 522.

[10] R. C. Coutinho, H. A. French, D. R. Selviah, D. Wickramasinghe, and H. D. Griffiths, "Detection of coherent light in an incoherent background," in Proc. Annu. Meeting IEEE LEOS, San Francisco, CA, 1999, vol. 1, pp. $247-248$.

[11] R. C. Coutinho, D. R. Selviah, and H. A. French, "Detection of partially coherent optical emission sources," in Proc. SPIE, D. P. Casasent and T.-H. Chao, Eds, 2000, vol. 4043, pp. 238-248.

[12] R. C. Coutinho, D. R. Selviah, R. F. Oulton, J. W. Gray, P. N. Stavrinou, H. D. Griffiths, and G. Parry, "Variable numerical aperture temporal coherence measurement of resonant cavity LED's," IEEE J. Lightw. Technol., vol. 21, no. 1, pp. 149-154, Jan. 2003.

[13] P. Sutton, "A novel optical pre-detector signal processing technique," Ph.D. dissertation, Univ. Southampton, Southampton, U.K., 1982.

[14] R. C. Coutinho, "High sensitivity optical detection using temporal coherence interferogram phase changes," Ph.D. dissertation, Univ. London, London, U.K., Jan. 2003. 
[15] J. B. Montgomery, R. B. Sanderson, and F. O. Baxley, "Tactical midinfrared background suppression in heavy clutter environments," in Proc. SPIE, O.E. Drummond, Ed, 2000, vol. 4048, Signal and Data Processing of Small Targets 2000, pp. 23-34.

[16] M. T. Eismann and C. R. Schwarz, "Focal plane array nonlinearity and nonuniformity impacts to target detection with thermal infrared imaging spectrometers," in Proc. SPIE, O.L Drummond, Ed, 1997, vol. 3063, Signal and Data Processing of Small Targets 2000 , pp. 164-173.

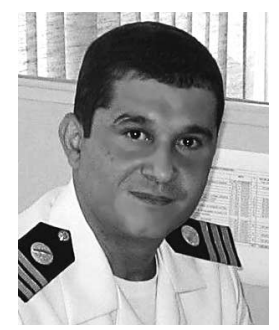

Ricardo C. Coutinho (M'99) received the Navy Officer diploma from the Brazilian Naval Academy, Rio de Janeiro, Brazil, in 1985, the M.Eng. degree in electronic engineering from the University of São Paulo, São Paulo, Brazil, in 1991, and the Ph.D. degree from the University College London, London, U.K., in 2003.

He was a Fleet Officer for two years in a frigate and was with the Brazilian Navy's Electronic Repair Center for six years as the Head of the Sensors Group. He is currently a Specialist in electrooptics with the Brazilian Navy Weapon Systems Directorate, Rio de Janeiro. He is also a Lecturer with the Department of Electronic Engineering, Federal Center of Technological Education, Rio de Janeiro. His research interests include the coherence properties of light, interferometric detection systems, testing and modeling of electrooptical systems, and infrared countermeasures.

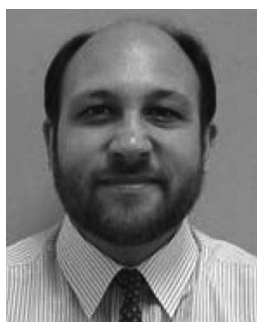

David R. Selviah (M'01) received the B.A. and M.A. degrees in physics and theoretical physics from Trinity College, Cambridge University, Cambrigde, U.K., in 1980 and 1983, respectively.

$\mathrm{He}$ was with the Allen Clark Research Center, Plessey (Caswell) Ltd., Oxfordshire, U.K. (now Bookham Technology Ltd.) for three years. He then joined the Department of Engineering Science, Oxford University, Oxford, U.K., where he was a Research Fellow for more than three years. In 1987, he joined the Department of Electronic and Electrical Engineering, University College London (UCL), London, U.K. where he is currently a Senior Lecturer. He has more than 100 publications of various types. His previous research interests include surface acoustic wave devices such as convolvers for phase-shift keying (PSK) pattern recognition, reflective array compressors for radar pulse compression, and PSK-to-minimum-shift keying (MSK) conversion filters. His current research interests include multimode polymer waveguide optical printed circuit boards, liquid-crystal display backlights, and real-time image processing.

Mr. Selviah is a member of the Institute of Physics, the Optical Society of America, and the European Optical Society.

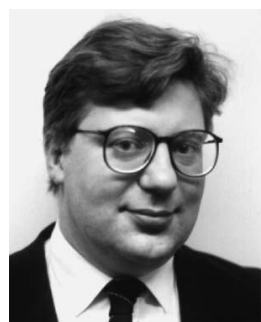

Hugh D. Griffiths (M'86-SM'90-F'99) received the M.A. degree in physics from Oxford University, Oxford, U.K., in 1978 and the Ph.D. and D.Sc(Eng). degrees from University College London, London, U.K., in 1986, and 2000, respectively.

He was the Head of the Department of Electronic and Electrical Engineering, University College London, from 2001 to 2006 and was appointed Principal of the Defence College of Management and Technology, Cranfield University, Shrivenham, U.K., in 2006. His research interests include radar and sonar systems and signal processing and antennas and antenna measurements.

Dr. Griffiths was a recipient of the IEEE AES Nathanson Award and the IEE Mountbatten and Maxwell Premium Awards in 1996. He was elected to the Fellowship of the Royal Academy of Engineering in 1997. 